\title{
Clinical Study \\ Influence of Pelvic Tilt on Polyethylene Wear after Total Hip Arthroplasty
}

\author{
Taro Tezuka, Yutaka Inaba, Naomi Kobayashi, Hiroyuki Ike, So Kubota, \\ Masaki Kawamura, and Tomoyuki Saito
}

Department of Orthopaedic Surgery, Yokohama City University, Yokohama 236-004, Japan

Correspondence should be addressed to Yutaka Inaba; yute0131@med.yokohama-cu.ac.jp

Received 23 April 2015; Accepted 6 July 2015

Academic Editor: George Babis

Copyright (c) 2015 Taro Tezuka et al. This is an open access article distributed under the Creative Commons Attribution License, which permits unrestricted use, distribution, and reproduction in any medium, provided the original work is properly cited.

\begin{abstract}
We aimed to evaluate the effects of pelvic tilt on polyethylene wear after total hip arthroplasty (THA). A total of 105 joints treated with primary THA were included; conventional polyethylene (CPE) liners were used in 43 hips and highly cross-linked polyethylene (HXLPE) liners were used in the remaining 62 hips. The pelvis was tilted $6^{\circ}$ posteriorly in the standing position as compared to the supine position, which resulted in significant increases of $1.7^{\circ}$ and $2.8^{\circ}$ in cup inclination in the CPE and HXLPE groups, respectively. Moreover, the change in pelvic tilt resulted in significant increases of $3.6^{\circ}$ and $4.9^{\circ}$ in cup anteversion in the CPE and HXLPE groups, respectively. For the CPE group, multiple regression analysis showed a significant association between the angle of pelvic tilt (PTA) and cup inclination and the polyethylene wear ratio. The adjusted $R^{2}$ of the regression model was larger for measures obtained in the standing position as compared to the supine position. For the HXLPE group, there was no significant relationship between radiographic parameters and polyethylene wear. Close observation of polyethylene wear is recommended for patients with severe posterior pelvic tilt who have undergone THA with conventional polyethylene.
\end{abstract}

\section{Introduction}

Inclination and anteversion of the cup in total hip arthroplasty (THA) affect clinical outcomes, including postoperative hip joint range of motion, risk of postoperative dislocation, and increased polyethylene wear, which can lead to osteolysis around the implant and cause loosening [1-3].

An increase in the posterior tilt position of the pelvis after THA has recently been reported $[4,5]$. A number of studies have investigated the relationship between pelvic tilt and the angle of the cup of the THA [6-8]. However, the specific relationship between pelvic tilt and polyethylene wear has not been investigated. Therefore, our study addressed two specific goals. The first was to quantify pelvic tilt after THA in the supine and standing positions and to evaluate the relationship of postural change in pelvic tilt with the angle of the cup. The second was to use multiple regression analysis to evaluate the relationship of pelvic tilt and the angle of the cup with polyethylene wear of the THA.

\section{Materials and Methods}

This study was a retrospective evaluation of patients who underwent primary THA at a single center, between 1986 and 2006. The methods and procedures were approved by the authors' institutional review board. Outcomes of a total of 105 joints, from 96 patients, were evaluated. Conventional polyethylene liners were used for 43 hips in 34 patients (CPE group), and highly cross-linked polyethylene liners were used in the other 62 hips from 62 patients (HXLPE group). The average age at surgery was $59 \pm 6.7$ years in the CPE group and $64 \pm 8.7$ years in the HXLPE group; there were no significant differences in age between the groups ( $p=0.56$, unpaired Student's $t$-test). The average follow-up period was $16.4 \pm 3.4$ years for the CPE group and $5.7 \pm 2.2$ years for the HXLPE group ( $p<0.01$; unpaired Student's $t$-test).

The porous coated anatomic THA system (Stryker Howmedica Osteonics Corp., Allendale, NJ), with a highdensity polyethylene liner (1050 GUR sterilized with gamma 
radiation in air) and a $26 \mathrm{~mm}$ femoral head component, was used for all hips in the CPE group. For the HXLPE group, the Secure Fit stem, Super Secure Fit stem, or CentPiller stem systems (Stryker Orthopaedics, Mahwah, NJ, USA) were used for 46 hips, and the VerSys fiber metal midcoat stem system (Zimmer International, Warsaw, IN, USA) was used for 16 hips. Forty-six Trident AD cups (Stryker Orthopaedics) and 16 Trilogy cups (Zimmer International) were used as cups, and 46 Crossfire liners (1050 GUR, which was irradiated by $10 \mathrm{Mrad}$ radiation and annealed polyethylene, Stryker Orthopaedics) and 16 Longevity liners (1050 GUR, which was 10 Mrad electron beam-irradiated and melted, Zimmer International) were used as polyethylene liners. A $26 \mathrm{~mm}$ femoral head was used in all cases.

For all cases, anterior-posterior (AP) pelvic radiographs were obtained in the supine and standing positions at the final follow-up session. The angle of inclination and degree of anteversion of the cup and the pelvic tilt angle (PTA) were measured from both supine and standing position radiographs. Polyethylene wear was also measured from the radiographs in the supine position. Anteversion of the cup was measured using the methods of Lewinnek [1]. The minor axis of the cup was designated as $D_{1}$ and the major axis was designated as $D_{2}$ on AP pelvic radiographs, and the angle of anteversion $(\alpha)$ was calculated as $\alpha=\sin ^{-1} D_{1} / D_{2}+5^{\circ}$. The PTA was calculated using the method of Doiguchi $[5,9]$. The lateral diameter $(T)$ and longitudinal diameter $(L)$ of the pelvic cavity were measured on AP pelvic radiographs, and the PTA was calculated as $-67 \times L / T+55.7^{\circ}$ for men and $-69 \times$ $L / T+61.6^{\circ}$ for women. According to this method, the larger the PTA, the more the pelvis tilts posteriorly, with a mean PTA of $20^{\circ}$ reported for healthy females. Linear polyethylene wear was measured using Roentgen Monographic Analysis (Roman) version 1.70 (Institute of Orthopaedics, Oswestry, UK) [10], following the method of Livermore et al. [11]. With the Roman software, 8 points on the edge of the cup were chosen and averaged to generate an edge and center. This process was repeated for the femoral head, and the displacement vector between the centers of the femoral head and cup components was measured. To rule out deformation of the polyethylene liner immediately after surgery, the difference in the central position of the femoral head between the 1-year postoperative and the final follow-up time points was measured [12]. The polyethylene wear rate after 1 year was determined by fitting a regression line to the data points at 1 year and at the final follow-up. The annual rate of wear (mm/year) was quantified as the slope of the regression line.

The change in PTA measured in the supine and standing positions was defined as $\triangle \mathrm{PTA}$; the change in the inclination and anteversion of the cup was defined, in a similar manner, as $\Delta$ inclination and $\Delta$ anteversion, respectively. Simple regression analysis was performed to analyze the relationships between the $\triangle \mathrm{PTA}$ and $\triangle$ inclination and between $\triangle \mathrm{PTA}$ and $\Delta$ anteversion in both THA groups. Additionally, Pearson's correlation coefficients were calculated to evaluate the association between the polyethylene wear rate and radiographic measurements after THA. Independent determinants of the polyethylene wear rate were identified by multiple linear
TABLE 1: Pelvic tilt angle (PTA) and the angle of inclination and anteversion of the cup in the supine and standing positions.

\begin{tabular}{lcccc}
\hline & CPE group & $p$ & HXLPE group & $p$ \\
\hline PTA $\left(^{\circ}\right)$ & & & & \\
$\quad$ Supine & $24.7 \pm 7.5$ & & $24.0 \pm 8.3$ & \\
$\quad$ Standing & $31.6 \pm 9.7$ & & $30.3 \pm 10.2$ & $<0.01$ \\
Cup inclination $\left(^{\circ}\right)$ & & & & \\
$\quad$ Supine & $40.2 \pm 6.7$ & & $41.6 \pm 6.6$ & $<0.01$ \\
$\quad$ Standing & $41.9 \pm 7.1$ & & $44.4 \pm 6.5$ & \\
Cup anteversion $\left(^{\circ}\right)$ & & & & \\
$\quad$ Supine & $14.6 \pm 5.5$ & & $20.0 \pm 8.8$ & $<0.01$ \\
$\quad$ Standing & $18.2 \pm 6.3$ & $<0.01$ & $24.9 \pm 9.7$ & \\
\hline
\end{tabular}

Unpaired Student's $t$-test.

regression. The radiographic measurements were entered simultaneously. The adjusted coefficient of multiple determination (adjusted $R^{2}$ ) was used to indicate the extent to which the variability in the polyethylene wear rate was accounted for by the independent variables. Standardized regression coefficients $(\beta)$ and associated $p$ values were determined to assess statistical significance $(p<0.05)$. All statistical analyses were performed using SPSS version 21.0 (IBM Corp., Armonk, NY, USA).

\section{Results}

In the CPE group, the PTA was $24.7 \pm 7.5^{\circ}$ in the supine position and $31.6 \pm 9.7^{\circ}$ in the standing position. From the supine to standing positions, the cup inclination changed from $40.2 \pm 6.7^{\circ}$ to $41.9 \pm 7.1^{\circ}(p<0.01)$, and cup anteversion changed from $14.6 \pm 5.5^{\circ}$ to $18.2 \pm 6.3^{\circ}(p<0.01)$. The PTA in the HXLPE group in the standing and supine positions were similar to those of the CPE group, with measures of 24.0 $\pm 8.3^{\circ}$ and $30.3 \pm 10.2^{\circ}$, respectively. There was a significant $6.3^{\circ}$ posterior tilt of the pelvis in the standing position as compared to that in the supine position (Figure 1). From the supine to standing positions, the cup inclination increased from $41.6 \pm 6.6^{\circ}$ to $44.4 \pm 6.5^{\circ}$, and cup anteversion increased from $20.0 \pm 8.8^{\circ}$ to $24.9 \pm 9.7^{\circ}$, indicating a significant increase in the values from the standing position to the supine position $(p<0.01, p<0.01)$ (Table 1$)$.

Figure 2 shows the results of the simple regression analysis between $\triangle \mathrm{PTA}$ and $\triangle$ inclination for the CPE and HXLPE groups. In both groups, there was a positive correlation between $\triangle$ PTA and $\Delta$ inclination (CPE group: $r=0.67, p<$ 0.01 ; HXLPE group: $r=0.43, p=0.02$ ). Based on the gradient of the graph, a $10^{\circ}$ posterior tilt of the pelvis was associated with an increase in inclination of $3^{\circ}$.

Figure 3 shows the results of simple regression analysis between $\triangle \mathrm{PTA}$ and $\triangle$ anteversion for the CPE and HXLPE groups. In both groups, there was a positive correlation between $\triangle \mathrm{PTA}$ and $\triangle$ anteversion (CPE group: $r=0.74$, $p<0.01$; HXLPE group: $r=0.82, p<0.01)$. Based on the gradient of the graph, a $10^{\circ}$ posterior tilt of the pelvis was associated with an increase in anteversion of $7^{\circ}$. 


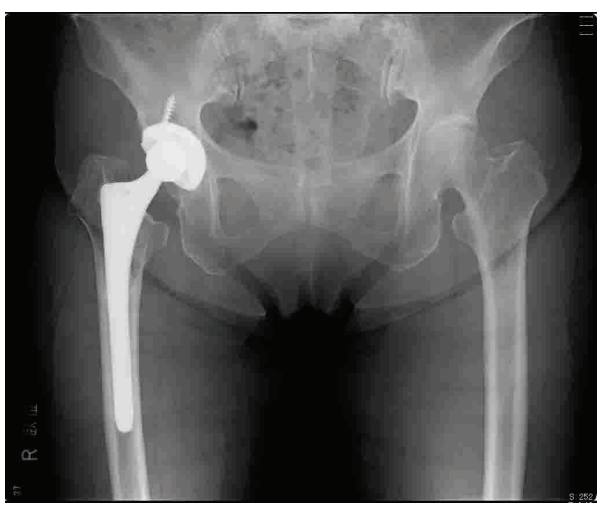

(a)

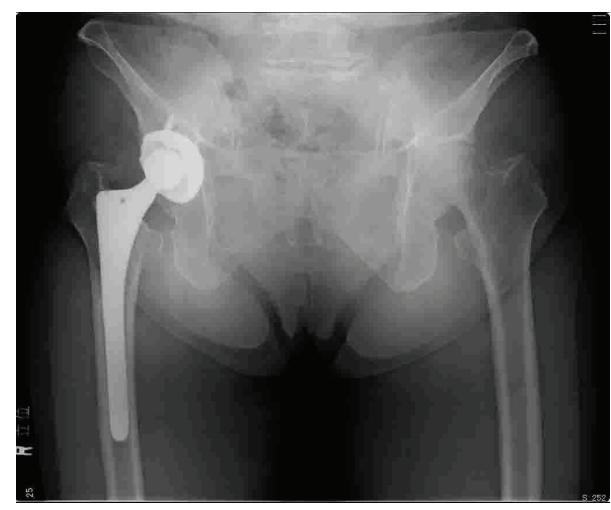

(b)

FIGURE 1: Radiographs of a 72-year-old woman treated with right hip THA. An HXLPE liner was implanted, and the annual polyethylene wear rate was $0.008 \mathrm{~mm} /$ year. (a) AP pelvic radiograph in the supine position 5 years after THA; cup inclination was $46^{\circ}$, cup anteversion was $16^{\circ}$, and PTA was $31^{\circ}$. (b) AP pelvic radiograph in the standing position shows that the pelvis is tilted posteriorly. The PTA was $59^{\circ}$, leading to an increase in cup inclination and anteversion; moreover, cup inclination was $52^{\circ}$ and cup anteversion was $38^{\circ}$.

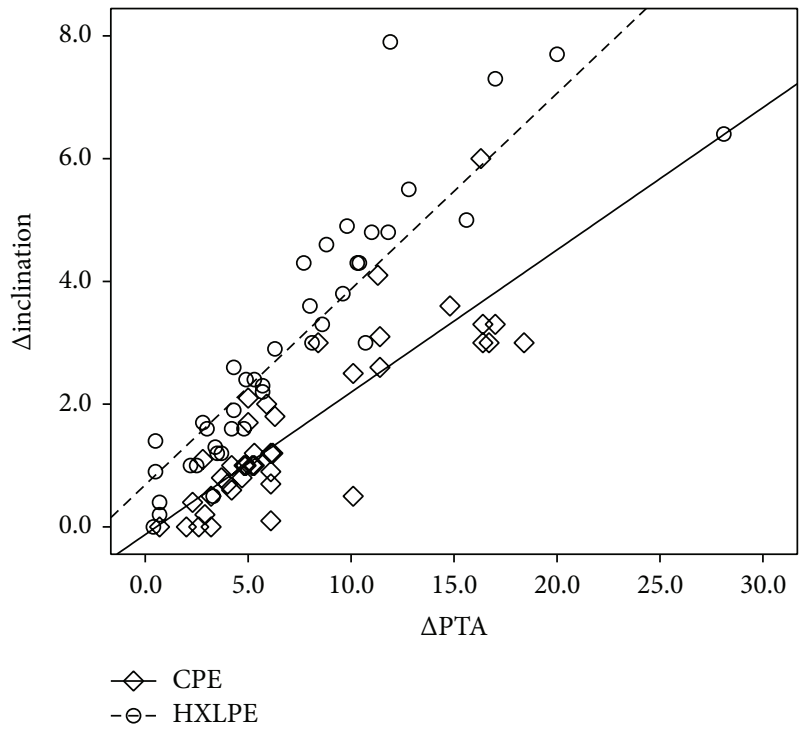

FIGURE 2: Simple linear regression between $\triangle \mathrm{PTA}$ and $\triangle$ inclination. In both groups, there was a positive correlation between $\triangle \mathrm{PTA}$ and $\Delta$ inclination (CPE group: $r=0.67 ; p<0.01$; HXLPE group: $r=0.43 ; p=0.02$ ). For every $10^{\circ}$ of pelvis tilt, the cup inclination increased by $3^{\circ}$.

The rate of polyethylene wear was $0.21 \pm 0.11 \mathrm{~mm} /$ year in the CPE group and $0.014 \pm 0.05 \mathrm{~mm}$ /year in the HXLPE group, and there was a significant difference in this value between the groups ( $p<0.01$; unpaired Student's $t$-test) (Figure 4).

Pearson's correlation coefficients between the rate of polyethylene wear and radiographic parameters in the supine and standing positions are reported in Table 2. For the CPE group, PTA in the standing position and cup inclination and anteversion in the supine and standing positions significantly influenced the rate of polyethylene wear. However, there was no significant relationship between polyethylene wear rate

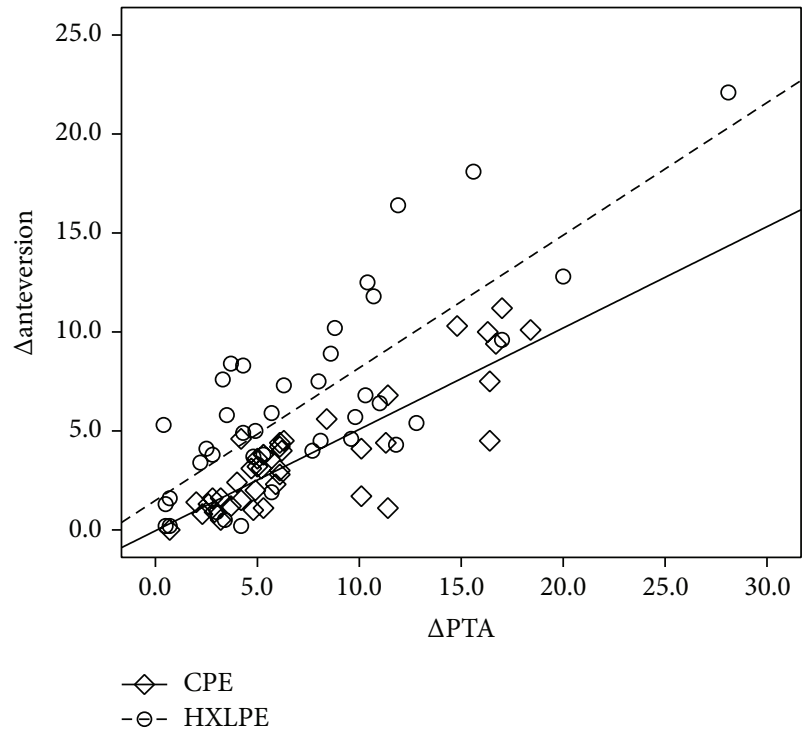

FIGURE 3: Simple linear regression between $\triangle P T A$ and $\Delta$ anteversion. In both groups, there was a positive correlation between $\triangle \mathrm{PTA}$ and $\Delta$ anteversion (CPE group: $r=0.74 ; p<0.01$; HXLPE group: $r=0.82 ; p<0.01)$. For every $10^{\circ}$ of pelvis tilt, the cup anteversion increased by $7^{\circ}$.

and these radiographic parameters for the HXLPE group. There was no significant correlation between $\triangle \mathrm{PTA}$ and annual rate of polyethylene wear in both groups.

For the CPE group, multiple regression analysis showed that the PTA (standard regression coefficient $[\beta]=0.292$; $p=0.04)$ and cup inclination angle $(\beta=0.398, p<0.01)$ were significantly associated with the polyethylene wear rate in the supine position. The final model accounted for $9 \%$ (adjusted $R^{2}=0.09$ ) of the variance in polyethylene wear rate. In the standing position, as in the supine position, multiple regression analysis showed that the PTA $(\beta=0.388 ; p=$ $0.03)$ and cup inclination angle $(\beta=0.417, p<0.01)$ were 
TABLE 2: Pearson's correlation coefficients between polyethylene wear rate and factors of pelvic tilt angle (PTA) and the angle of inclination and anteversion of the cup.

\begin{tabular}{lccccc}
\hline \multirow{2}{*}{ Parameter } & \multirow{2}{*}{ Position } & \multicolumn{2}{c}{ CPE group } & \multicolumn{2}{c}{ HXLPE group } \\
& & $r$ & $p$ & $r$ & $p$ \\
\hline \multirow{2}{*}{ PTA } & Supine & 0.24 & 0.11 & 0.21 & 0.06 \\
& Standing & 0.34 & 0.02 & 0.1 & 0.36 \\
\hline \multirow{2}{*}{ Cup inclination } & Supine & 0.39 & $<0.01$ & 0.27 & 0.16 \\
& Standing & 0.43 & $<0.01$ & 0.07 & 0.53 \\
\hline \multirow{2}{*}{ Cup anteversion } & Supine & 0.23 & 0.02 & 0.18 & 0.1 \\
& Standing & 0.37 & 0.02 & 0.09 & 0.93 \\
\hline \multirow{2}{*}{ PTA } & & 0.29 & 0.06 & 0.18 & 0.38 \\
\hline
\end{tabular}

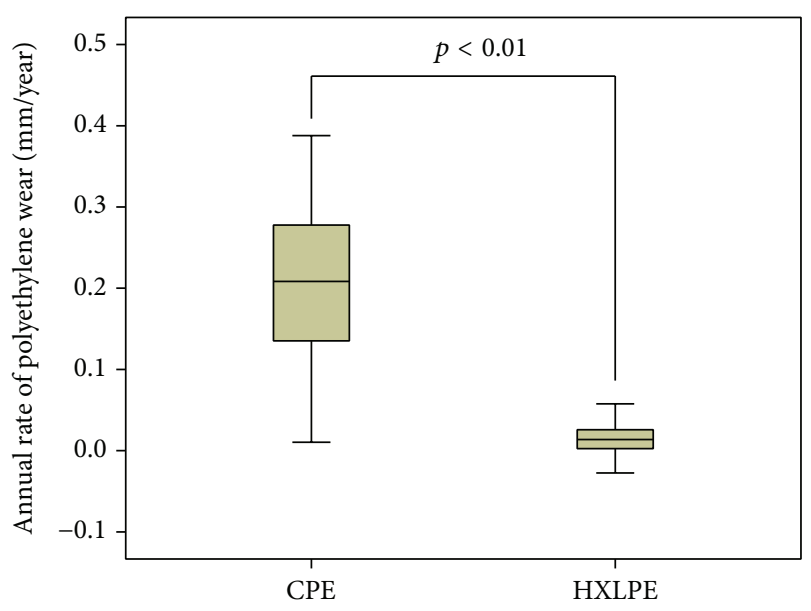

FIgURE 4: The annual polyethylene rate after THA. The polyethylene annual wear rate was $0.21 \pm 0.10 \mathrm{~mm} /$ year for the CPE group and $0.014 \pm 0.05 \mathrm{~mm} /$ year for the HXLPE group; there was a significant difference in this value between the groups (unpaired Student's $t$ test).

significantly associated with the polyethylene wear rate and the final model accounted for $16 \%$ (adjusted $R^{2}=0.16$ ) of the variance in the polyethylene wear rate (Table 3). For the HXLPE group, there was no significant correlation between the annual polyethylene linear wear rate and radiographic parameters (Table 4).

\section{Discussion}

It is well known that polyethylene linear wear is one of the causes of aseptic loosening leading to THA revision. Several factors that affect polyethylene wear have been reported, such as the size of the femoral head; the thickness of the polyethylene liner [13]; patient age [14], BMI [15], and postoperative activities of daily living $[13,14]$; position of the cup [16]; and cup inclination and offset [17]. To our knowledge, our study is the first to assess the association between polyethylene wear and pelvic tilt.

Patil et al. [18] evaluated the contact stress of the hip joint by finite-element analysis and reported a concomitant increase in contact stress on the joint surface with the increase in the angle of inclination of the cup. Conversely, contact stress decreased as the angle of anteversion of the cup increased posterior tilting of the pelvis increased both the inclination and anteversion of the cup. Therefore, we were mainly concerned about the manner in which pelvic tilt influences polyethylene wear.

From the results of our current study, both inclination and anteversion of the cup increased through the posterior tilt of the pelvis after THA. Cup inclination and anteversion increased by $3^{\circ}$ and $7^{\circ}$, respectively, with a $10^{\circ}$ posterior tilt of the pelvis. Lembeck et al. [19] reported that each degree of pelvic tilt requires a $0.7^{\circ}$ correction in the angle of anteversion of the acetabular cup. Babisch et al. [20] also reported that cup inclination changed by approximately $0.3^{\circ}$ and cup anteversion changed by approximately $0.8^{\circ}$ per $1^{\circ}$ change of pelvic tilt. Our clinical results were consistent with the results of these modeling studies and, therefore, were considered to be appropriate.

For the CPE group, the annual polyethylene liner wear rate correlated with cup inclination and pelvic tilt angle. Cup anteversion did not correlate with polyethylene wear rate and, therefore, was not considered to be a major factor influencing polyethylene wear. Multivariate regression analysis in the present study indicated that pelvic tilt and cup inclination were significant factors for polyethylene wear, and the current study is the first to report the influence of pelvic tilt on polyethylene wear after THA. The adjusted $R^{2}$ values for pelvic tilt and cup inclination were larger in the standing position than in the supine position; hence, the radiographic parameters in the standing position are better indicators of the influence of polyethylene wear as compared to those in the supine position.

For the HXLPE group, although inclination and anteversion increased through an increase in posterior tilt of the pelvis after THA, we could not find any significant correlation between polyethylene annual linear wear rate and these parameters. We believe that this is largely due to the improvement of the quality of polyethylene liner. However, considering the results from modeling studies described above, contact stress might increase as a function of increased cup inclination in cases with severe pelvic tilt after THA in this group as well.

Another concern was whether the change in pelvic tilt from supine to standing position (i.e., $\triangle \mathrm{PTA}$ ) influences polyethylene wear. We identified some cases where the amount of change in pelvic tilt from the supine to standing positions was large. Initially, we thought that such large changes in pelvic tilt might be one of the factors influencing polyethylene wear; however, we did not find the amount of postural change in pelvic tilt to be a significant factor of the annual rate of polyethylene wear.

The limitations of this study should be considered. First, due to the retrospective nature of this study, the follow-up period for the HXLPE group was significantly shorter than for the CPE group. Dai et al., who followed up patients with THA for a period of $131 \pm 10$ months, reported that the migration of the femoral head at an average period of 3.4 months after THA accounted for $56 \%$ of the total migration of the femoral 
TABLE 3: Multiple regression analysis between conventional polyethylene wear rate and factors of pelvic tilt angle (PTA) and the angle of inclination and anteversion of the cup.

\begin{tabular}{llccrc}
\hline Position & Parameter & Adjusted $R^{2}$ & Regression coefficient & Standard regression coefficient $(\beta)$ & 0.292 \\
\multirow{3}{*}{ Supine } & PTA & & 0.004 & 0.398 & 0.04 \\
& Cup inclination & 0.09 & 0.005 & 0.03 & 0.01 \\
& Cup anteversion & & 0.02 & 0.388 & 0.03 \\
\multirow{3}{*}{ Standing } & PTA & & 0.002 & 0.417 & $<0.01$ \\
& Cup inclination & 0.16 & 0.002 & 0.085 & 0.59 \\
\hline
\end{tabular}

TABLE 4: Multiple regression analysis between highly cross-linked polyethylene wear rate and factors of pelvic tilt angle (PTA) and the angle of inclination and anteversion of the cup.

\begin{tabular}{lcccc}
\hline Position & Adjusted $R^{2}$ & $\begin{array}{c}\text { Regression } \\
\text { coefficient }\end{array}$ & $\begin{array}{c}\text { Standard } \\
\text { regression } \\
\text { coefficient }\end{array}$ & $p$ \\
\hline Supine & & -0.003 & -0.58 & 0.06 \\
$\quad$ PTA & 0.07 & 0.003 & 0.35 & 0.07 \\
Cup inclination & & -0.004 & -0.48 & 0.17 \\
$\quad$ Cup anteversion & & & & \\
\hline Standing & & -0.001 & -0.1 & 0.42 \\
PTA & & 0.001 & 0.06 & 0.63 \\
Cup inclination & 0.03 & -0.001 & -0.01 & 0.94 \\
Cup anteversion & & & & \\
\hline
\end{tabular}

component in the first 2 years and that the degree of wear in the first postoperative year accounted for nearly $40 \%$ of the total wear [12]. Dai et al. determined that after an early change in polyethylene, known as the creep response, the rate of wear decreased gradually with time and stabilized. In our study, we excluded the measures of wear over the first year after THA and compared the polyethylene wear over steady periods, with an average follow-up period of the HXLPE group of 5.7 years. Therefore, we do not believe that the difference in the follow-up period between the CPE and HXLPE groups influenced our results.

In our study, we also did not evaluate the change in pelvic tilt before and after THA, as preoperative pelvic radiographs in the supine and standing positions had not been obtained in a sufficient number of patients before THA. Therefore, the effect of the change of pelvic tilt after THA on polyethylene wear is unknown. Our results do show, however, that pelvic tilt at any time point after THA could be a factor influencing polyethylene wear. Close observation is recommended for patients with severe posterior pelvic tilt measures who have undergone THA using conventional polyethylene.

\section{Conclusion}

Compared to the supine position, there was a significant increase in posterior pelvic tilt in the standing position, resulting in increased cup inclination and anteversion. In THA with conventional polyethylene liners, pelvic tilt in the supine and standing positions correlated with the annual rate of polyethylene linear wear as well as with cup inclination. In HXLPE hips, pelvic tilt had no effect on the annual rate of wear of the liner.

\section{Conflict of Interests}

The authors declare no conflict of interests.

\section{Acknowledgments}

The authors wish to thank Dr. Yohei Yukizawa, Dr. Chie Aoki, Dr. Hiroshi Fujimaki, and Dr. Yasuhide Hirata for their valuable contribution to the current study.

\section{References}

[1] G. E. Lewinnek, J. L. Lewis, R. Tarr, C. L. Compere, and J. R. Zimmerman, "Dislocations after total hip-replacement arthroplasties," The Journal of Bone \& Joint Surgery Series A, vol. 60, no. 2, pp. 217-220, 1978.

[2] F. J. Kummer, S. Shah, S. Iyer, and P. E. DiCesare, "The effect of acetabular cup orientations on limiting hip rotation," The Journal of Arthroplasty, vol. 14, no. 4, pp. 509-513, 1999.

[3] R. Biedermann, A. Tonin, M. Krismer, F. Rachbauer, G. Eibl, and B. Stöckl, "Reducing the risk of dislocation after total hip arthroplasty," The Journal of Bone and Joint Surgery-British Volume, vol. 87, no. 6, pp. 762-769, 2005.

[4] B. Blondel, S. Parratte, P. Tropiano, V. Pauly, J.-M. Aubaniac, and J.-N. Argenson, "Pelvic tilt measurement before and after total hip arthroplasty," Orthopaedics \& Traumatology: Surgery \& Research, vol. 95, no. 8, pp. 568-572, 2009.

[5] N. Taki, N. Mitsugi, Y. Mochida, Y. Akamatsu, and T. Saito, "Change in pelvic tilt angle 2 to 4 years after total hip arthroplasty," Journal of Arthroplasty, vol. 27, no. 6, pp. 940-944, 2012.

[6] M. Tannast, U. Langlotz, K. A. Siebenrock, M. Wiese, K. Bernsmann, and F. Langlotz, "Anatomic referencing of cup orientation in total hip arthroplasty," Clinical Orthopaedics and Related Research, no. 436, pp. 144-150, 2005.

[7] M. Haenle, A. Heitner, W. Mittelmeier et al., "Assessment of cup position from plain radiographs: impact of pelvic tilting," Surgical and Radiologic Anatomy, vol. 29, no. 1, pp. 29-35, 2007.

[8] T. A. Kalteis, M. Handel, B. Herbst, J. Grifka, and T. Renkawitz, "In vitro investigation of the influence of pelvic tilt on acetabular cup alignment," The Journal of Arthroplasty, vol. 24, no. 1, pp. 152-157, 2009.

[9] Y. Doiguchi, K. Iwasaki, K. Yamada et al., "Correlation between pelvic inclination and radiological shape of the pelvic cavity," Seikei geka to saigai geka, vol. 41, no. 2, pp. 641-645, 1992. 
[10] C. H. Geerdink, B. Grimm, W. Vencken, I. C. Heyligers, and A. J. Tonino, "The determination of linear and angular penetration of the femoral head into the acetabular component as an assessment of wear in total hip replacement: a comparison of four computer-assisted methods," The Journal of Bone \& Joint Surgery-British Volume, vol. 90, no. 7, pp. 839-846, 2008.

[11] J. Livermore, D. Ilstrup, and B. Morrey, "Effect of femoral head size on wear of the polyethylene acetabular component," The Journal of Bone \& Joint Surgery Series A, vol. 72, no. 4, pp. 518$528,1990$.

[12] X. Dai, H. Omori, Y. Okumura et al., "Serial measurement of polyethylene wear of well-fixed cementless metal-backed acetabular component in total hip arthroplasty: an over 10 year follow-up study," Artificial Organs, vol. 24, no. 9, pp. 746-751, 2000.

[13] D. J. Berry, C. L. Barnes, R. D. Scott, M. E. Cabanela, and R. Poss, "Catastrophic failure of the polyethylene liner of uncemented acetabular components," Journal of Bone and Joint SurgeryBritish Volume, vol. 76, no. 4, pp. 575-578, 1994.

[14] A. P. D. Elfick, R. M. Hall, I. M. Pinder, and A. Unsworth, "Wear in retrieved acetabular components: effect of femoral head radius and patient parameters," The Journal of Arthroplasty, vol. 13, no. 3, pp. 291-295, 1998.

[15] D. L. Scott, P. A. Campbell, C. D. McClung, and T. P. Schmalzried, "Factors contributing to rapid wear and osteolysis in hips with modular acetabular bearings made of hylamer," The Journal of Arthroplasty, vol. 15, no. 1, pp. 35-46, 2000.

[16] K. Hirakawa, N. Mitsugi, T. Koshino, T. Saito, Y. Hirasawa, and T. Kubo, "Effect of acetabular cup position and orientation in cemented total hip arthroplasty," Clinical Orthopaedics and Related Research, no. 388, pp. 135-142, 2001.

[17] N. J. Little, C. A. Busch, J. A. Gallagher, C. H. Rorabeck, and R. B. Bourne, "Acetabular polyethylene wear and acetabular inclination and femoral offset," Clinical Orthopaedics and Related Research, vol. 467, no. 11, pp. 2895-2900, 2009.

[18] S. Patil, A. Bergula, P. C. Chen, C. W. Colwell Jr., and D. D. D'Lima, "Polyethylene wear and acetabular component orientation," The Journal of Bone \& Joint Surgery Series A, vol. 85, no. 4, pp. 56-63, 2003.

[19] B. Lembeck, O. Mueller, P. Reize, and N. Wuelker, "Pelvic tilt makes acetabular cup navigation inaccurate," Acta Orthopaedica, vol. 76, no. 4, pp. 517-523, 2005.

[20] J. W. Babisch, F. Layher, and L. P. Amiot, "The rationale for tiltadjusted acetabular cup navigation," The Journal of Bone and Joint Surgery-American Volume, vol. 90, pp. 357-365, 2008. 


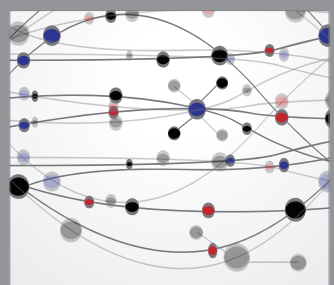

The Scientific World Journal
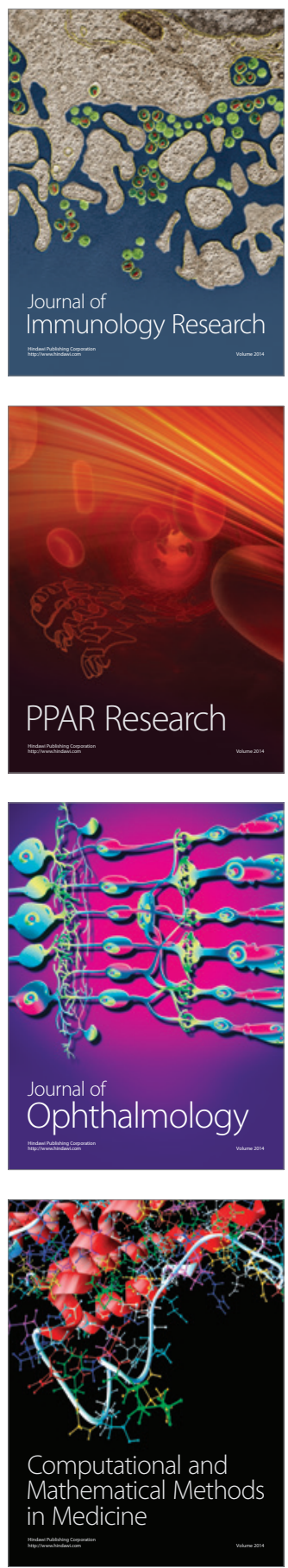

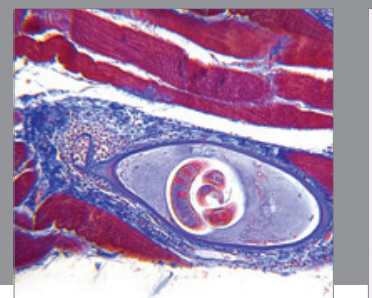

Gastroenterology

Research and Practice
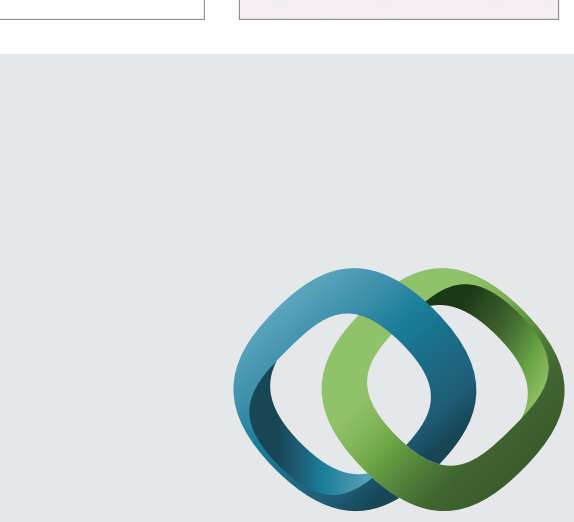

\section{Hindawi}

Submit your manuscripts at

http://www.hindawi.com
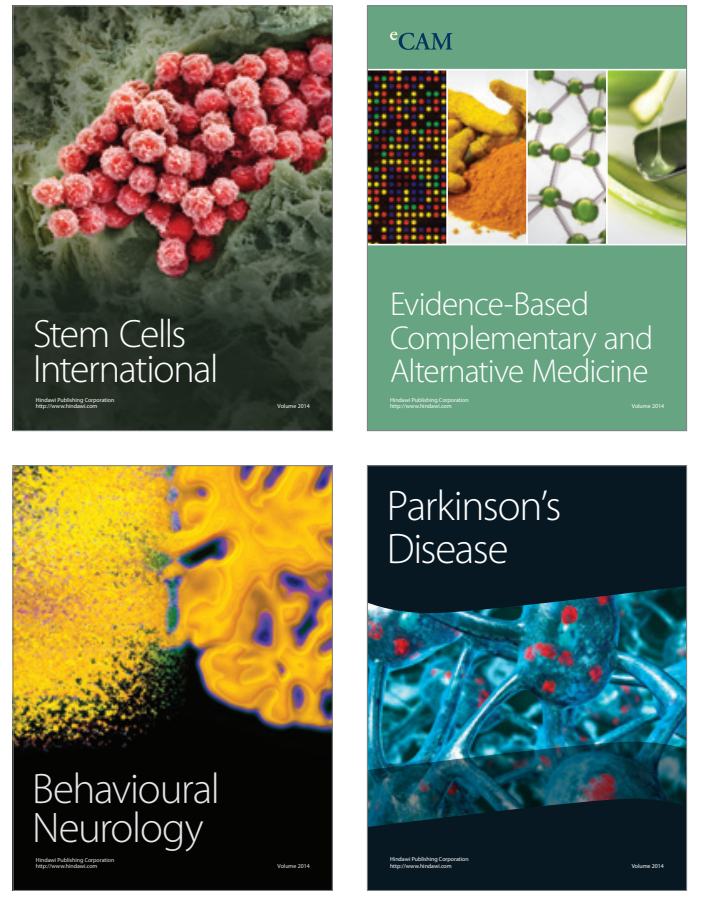
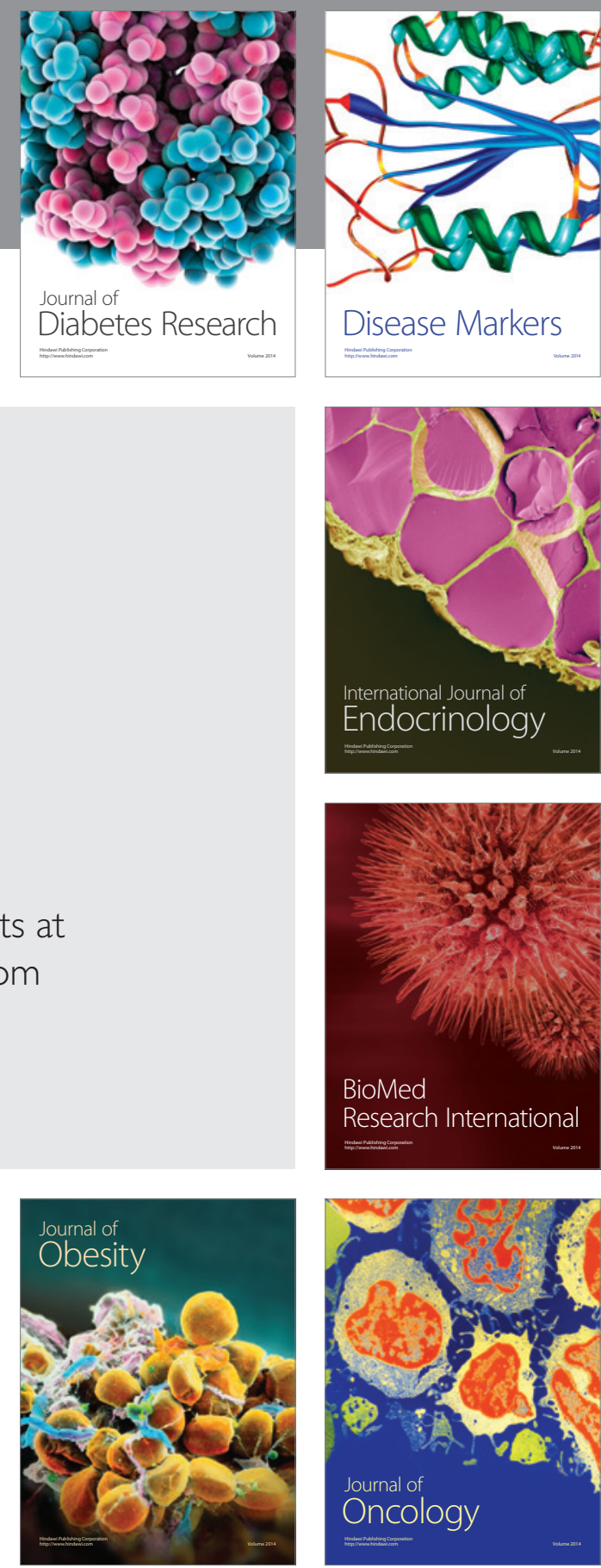

Disease Markers
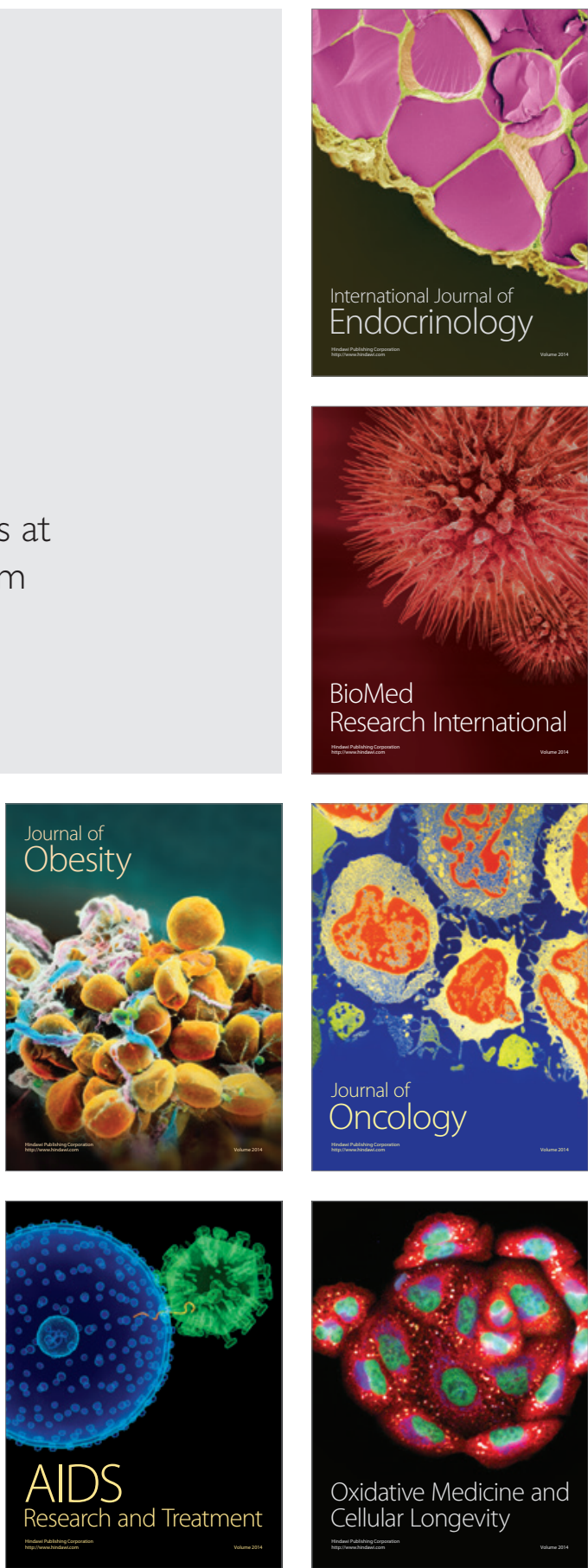